1Programa de Intervención Temprana, Instituto Psiquiátrico J. Horwitz Barak. Santiago, Chile. 2Departamento de Psiquiatría, Escuela de Medicina, Pontificia Universidad Católica de Chile. Santiago, Chile. ${ }^{3}$ Escuela de Medicina, Universidad Finis Terrae. Santiago, Chile. ${ }^{4}$ Clínica Psicológica, Universidad Diego Portales. Santiago, Chile. ${ }^{5}$ Departamento de Neurología y Psiquiatría, Facultad de Medicina, Clínica Alemana, Universidad del Desarrollo. Santiago, Chile. aPsicólogaClínica.

Los autores declararan no tener conflictos de interés.

Recibido el 11 de noviembre de 2019, aceptado el 2 de noviembre de 2020

Correspondencia a: Dr. Nicolás Crossley Diagonal Paraguay 362. Santiago, Chile. ncrossley@uc.cl.

\section{Uso de cannabis en jóvenes hospitalizados por un primer episodio de psicosis: un estudio caso-control}

\author{
CARMEN PAZ CASTAÑEDA ${ }^{1, a}$, LUZ MARÍA ALLIENDE ${ }^{2, a}$, \\ BÁRBARA IRURETAGOYENA ${ }^{2}$, RUBÉN NACHAR ${ }^{1,3}$, \\ FELIPE MANCILLA $^{1}$, CAMILA DÍAZ ${ }^{1}$, CARLOS GALLARDO ${ }^{1,4}$, \\ CRISTIÁN MENA ${ }^{1}$, JUAN PABLO RAMÍREZ-MAHALUF ${ }^{2}$, \\ JUAN UNDURRAGA ${ }^{1,5}$, ALFONSO GONZÁLEZ-VALDERRAMA ${ }^{1,3}$, \\ NICOLÁS A. CROSSLEY ${ }^{*}$
}

\section{Cannabis use among hospitalized young people experiencing a first episode of psychosis: a case control study}

Background: Cannabis use among young people in Chile has increased significantly in the last years. There is a consistent link between cannabis and psychosis. Aim: To compare cannabis use in patients with a first episode of psychosis and healthy controls. Material and Methods: We included 74 patients aged $20 \pm 3$ years ( $78 \%$ males) admitted to hospital with a first episode of psychosis and a group of 60 healthy controls aged $23 \pm 4$ years ( $63 \%$ males). Cannabis consumption was assessed, including age of first time use and length of regular use. Results: Patients with psychosis reported a non-significantly higher frequency of life-time cannabis use. Patients had longer periods of regular cannabis use compared with healthy subjects (Odds ratio [OR] 2.4; 95\% confidence intervals [CI] 1.14-5.05). Patients also used cannabis for the first time at an earlier age (16 compared with 17 years, $p<0.0$ ). The population attributable fraction for regular cannabis use associated with hospital admissions due to psychosis was $17.7 \%$ (95\% CI 1.2-45.5\%). Conclusions: Cannabis use is related to psychosis in this Chilean group of patients. This relationship is stronger in patients with early exposure to the drug and longer the regular use. One of every five admissions due to psychosis is associated with cannabis consumption. These data should influence cannabis legislation and the public policies currently being discussed in Chile.

(Rev Med Chile 2020; 148: 1606-1613)

Key words: Cannabis; Case-Control Studies; Psychotic Disorders.
E 1 uso de cannabis en Chile ha estado en el foco de la opinión pública en los últimos años. Esto debido al posible uso terapéutico de los derivados del cannabis ${ }^{1,2}$, así como también por las propuestas en torno a la despenalización del consumo recreacional de esta sustancia. Al mismo tiempo, la percepción de la población general del potencial daño por cannabis ha ido progresivamente descendiendo, fenómeno que no se ha manifestado con otras drogas como la cocaína o el alcohol ${ }^{3}$. Esta disminución en la percepción del riesgo de cannabis se ha asociado a un aumento progresivo del uso de esta sustancia en los últimos 8 años, especialmente en jóvenes 
entre 19 y 25 años, además de una reducción en la edad en que los jóvenes se exponen a la droga por primera vez ${ }^{3,4}$.

Varios estudios han mostrado que el consumo de cannabis se asocia a un aumento de la incidencia de psicosis en jóvenes, particularmente esquizofrenia. Esto fue sugerido inicialmente en una cohorte de conscriptos en Suecia, que permitió relacionar el nivel de exposición a cannabis con la hospitalización subsiguiente por un episodio psicótico. En esa cohorte, el riesgo de psicosis aumentó hasta 6 veces en aquellos sujetos con consumo previo mayor a 50 ocasiones, comparado con aquellos sin consumo ${ }^{5}$. Estudios posteriores han confirmado este hallazgo. Un metaanálisis reciente, que resume los principales estudios sobre el tema, sugirió que el riesgo de psicosis en aquellos jóvenes que han usado marihuana a lo largo de su vida es 3,90 veces mayor ${ }^{6}$. Sin embargo, existen voces disidentes que han sugerido la posibilidad de una causalidad inversa, en otras palabras, que los pacientes se automedicarían con cannabis para contrarrestar los síntomas psicóticos emergentes ${ }^{7}$. No obstante, la existencia de un riesgo elevado después de años de exposición a cannabis es difícil de explicar considerando esta perspectiva ${ }^{8}$.

El cannabis contiene múltiples compuestos con diferentes efectos en el cerebro, incluyendo tetrahidrocannabinol (THC) y cannabidiol (CBD). El THC se ha relacionado con la aparición de síntomas psicóticos ${ }^{9-11}$. Por otra parte, el CBD se ha asociado a un efecto ansiolítico y antipsicótico ${ }^{12,13}$. El riesgo de psicosis ha sido particularmente asociado con el uso de cannabis con altas concentraciones de $\mathrm{THC}^{14}$, esto es, concentraciones en torno a $15 \%$ de THC (marihuana de "alta potencia") ${ }^{15}$. La disponibilidad para consumo de este tipo de cannabis podría explicar las diferencias geográficas descritas en la incidencia de psicosis ${ }^{16}$.

Un estudio previo demostró una mayor prevalencia de trastornos psicóticos en pacientes en tratamiento por adicción a cannabis en $\mathrm{Chile}^{17}$, sugiriendo que el cannabis en Chile tendría un efecto importante sobre el riesgo a presentar psicosis. Sin embargo, no se pudo descartar que pacientes con consumo de cannabis y psicosis sean derivados a atención de salud con mayor frecuencia que aquellos con consumo y sin psicosis. Considerando el potencial impacto que podría tener el aumento del consumo de cannabis en nuestros jóvenes, presentamos un estudio caso-control, cuyo objetivo es establecer la asociación entre presencia de psicosis y consumo de cannabis local, y así contribuir a la discusión sobre cannabis y su regulación en Chile.

\section{Métodos}

Este es un estudio caso-control realizado entre diciembre de 2016 y septiembre de 2018. Todos los jóvenes hospitalizados con un primer episodio psicótico en la Unidad de Primer Episodio Psicótico (Sector 1) del Instituto Psiquiátrico Dr. José Horwitz Barak fueron invitados a participar en este estudio. Esta unidad es parte del Programa de Intervención Temprana en Psicosis (ProITP) del Instituto Psiquiátrico ${ }^{18}$ y recibe jóvenes atendidos dentro del sistema público de referencia nacional entre 18 y 25 años con psicosis. Para ser incluidos, los jóvenes debían tener capacidad de consentir a la participación, además de cumplir criterios para alguno de los siguientes trastornos evaluados según la entrevista neuropsiquiátrica Mini International Neuropsychiatric Interview (M.I.N.I. ${ }^{19}$ : esquizofrenia, trastorno esquizofreniforme, trastorno delirante, psicosis breve, manía psicótica, depresión psicótica. A su vez, se reclutó un grupo control, incluyendo amigos de los pacientes, que no cumplían criterios diagnósticos para enfermedad psiquiátrica activa según M.I.N.I y sin historia de psicosis previa.

Datos demográficos, incluyendo edad, sexo y comuna de residencia, fueron obtenidos a través de una entrevista semiestructurada con los pacientes y sus familiares.

Nuestro interés era evaluar la exposición histórica a cannabis. Para ello, se aplicó el cuestionario Cannabis Experience Questionnaire ${ }^{20}$. Nos enfocamos en la evaluación histórica de grado de consumo del cuestionario, particularmente edad de primera exposición y duración de consumo regular (definido en el cuestionario como uso de cannabis al menos $1 \mathrm{vez}$ al mes). No se evaluó el consumo reciente con exámenes de orina para detectar sustancias, ya que nuestro enfoque estaba en el consumo histórico. Se obtuvieron, además, datos sobre consumo en vida de alcohol, cocaína o pasta base, y otras drogas (hipnosedantes, LSD, ayahuasca, éxtasis).

Para el análisis estadístico de la temporalidad se utilizaron tests no paramétricos debido a la no 
normalidad de este tipo de datos, o bien tests de $\chi^{2}$ para evaluar frecuencias. Se calcularon odds ratios, que fueron ajustados por variables confundentes usando el procedimiento de Mantel-Haenszel ${ }^{21}$. La fracción atribuible poblacional a cannabis fue calculada mediante la siguiente fórmula ${ }^{22}$ :

$$
R P A=100 * \frac{P_{e}^{\star}(O R-1)}{1+\left(P_{e}^{\star}[O R-1]\right)}
$$

donde $P_{e}$ es la prevalencia de la exposición a cannabis, y OR son los odds ratios calculados. $\mathrm{p}<0,05$ fue considerado estadísticamente significativo.

El estudio fue aprobado por el Comité de Ética del Servicio de Salud Metropolitano Norte y el de la Pontificia Universidad Católica de Chile.

\section{Resultados}

\section{Comparación entre jóvenes hospitalizados y controles}

Se incluyó un total de 134 sujetos, que consintieron a participar en nuestro estudio. De estos, 74 presentaban un primer episodio psicótico y 60 eran controles. Como se observa en la Tabla 1, la mayoría de los jóvenes hospitalizados eran hombres $(78,4 \%)$ y presentaban un episodio de psicosis no afectiva $(87,8 \%)$. Los pacientes fueron reclutados en etapa aguda durante su hospitalización, con una mediana de días de estadía al momento de la evaluación de 19 días. La Figura 1 muestra la procedencia de los jóvenes hospitalizados, resaltando que la residencia de ellos estaba distribuida

Tabla 1. Descripción de la muestra

\begin{tabular}{|lccl|}
\hline & $\begin{array}{c}\text { Pacientes } \\
(\mathbf{n}=\mathbf{7 4 )}\end{array}$ & $\begin{array}{c}\text { Controles } \\
(\mathbf{n}=\mathbf{6 0})\end{array}$ & Comparación \\
\hline Edad promedio (DE) & $20,3(3,53)$ & $23,0(3,58)$ & $\mathrm{P}<0,00002$, estadístico t-test $-4,45$ (gdl $=132)$ \\
\hline \% Hombres & $78,4 \%$ & $63,3 \%$ & $\mathrm{p}=0,055$, estadístico de $\chi^{2} 3,69, \mathrm{gdl}=1$ \\
\hline \% Psicosis no afectiva & $87,8 \%$ & - & $\mathrm{NA}$ \\
\hline $\begin{array}{l}\text { Días hospitalización mediana } \\
\text { (rango intercuartil) }\end{array}$ & 19 días $(14-29)$ & - & $\mathrm{NA}$ \\
\hline
\end{tabular}

$\mathrm{DE}=$ desviación estándar; $\mathrm{NA}=$ no se aplica; $\mathrm{gdl}=$ grados de libertad.

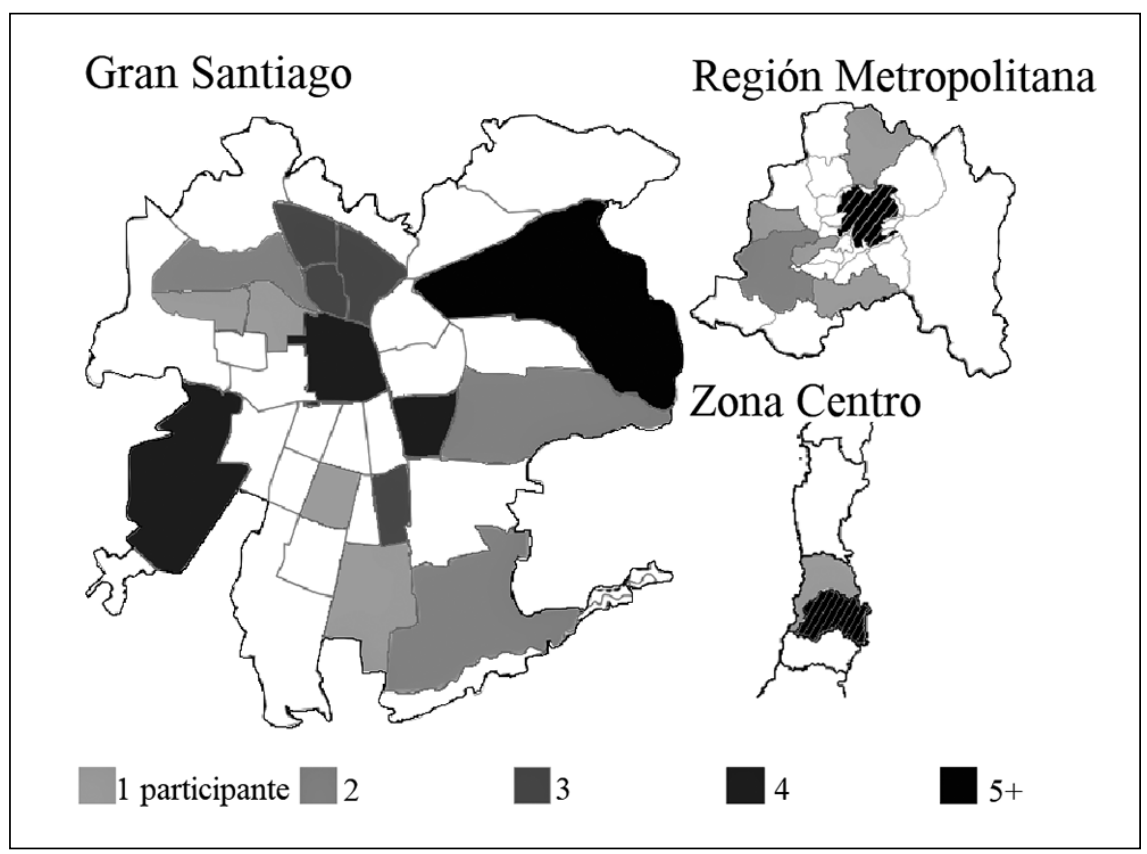

Figura 1. Comuna de origen de los participantes del Gran Santiago y la Región Metropolitana. Adicionalmente, se muestra la región de origen de participantes con residencia fuera de la Región Metropolitana. 
Tabla 2. Uso de sustancias en pacientes con psicosis y controles

\begin{tabular}{|c|c|c|c|}
\hline & Pacientes $(n=74)$ & Controles $(n=60)$ & Comparación \\
\hline$\%$ consumo cannabis vida & $87,8 \%$ & $85 \%$ & $\begin{array}{l}P=0,63 ; \text { estadística } \\
\chi^{2} 0,23 ; \text { gdl }=1\end{array}$ \\
\hline $\begin{array}{l}\text { \% consumo regular cannabis en la } \\
\text { vida }\end{array}$ & $77 \%$ & $58,3 \%$ & $\begin{array}{l}P=0,02, \text { estadística } \\
\chi^{2} 5,387, \text { gdl }=1\end{array}$ \\
\hline $\begin{array}{l}\text { Período de consumo regular-mediana } \\
\text { de meses y rango intercuartil } \\
\text { (N con datos completos reportados) }\end{array}$ & $\begin{array}{l}14 \text { meses, } \\
3-36 \text { riq } \\
(n=70)\end{array}$ & $\begin{array}{l}3 \text { meses, } \\
0-24 \text { riq } \\
(n=59)\end{array}$ & $\begin{array}{l}P=0,023 \text { prueba de } \\
\text { Mann-Whitney } U\end{array}$ \\
\hline $\begin{array}{l}\text { Edad primer consumo de cannabis } \\
\text { (mediana, riq) }\end{array}$ & $\begin{array}{l}16 \text { años (14-17; } N=64 \text { de } \\
65 \text { con consumo reportado } \\
\text { alguna vez en la vida) }\end{array}$ & $\begin{array}{l}17 \text { años }(16-18 ; N=51 \text { de } \\
51 \text { con consumo reportado } \\
\text { alguna vez en la vida) }\end{array}$ & $\begin{array}{l}P=0,004, \text { prueba de } \\
\text { Mann-Whitney } U\end{array}$ \\
\hline Consumo de alcohol - vida & $\begin{array}{l}65,3 \% \\
(n=72)\end{array}$ & $\begin{array}{l}74,1 \% \\
(n=58)\end{array}$ & $\begin{array}{l}P=0,28 ; \text { estadística } \\
\chi^{2} 1,18 ; \text { gdl }=1\end{array}$ \\
\hline $\begin{array}{l}\text { Consumo de cocaína/ } \\
\text { pasta base - vida }\end{array}$ & $\begin{array}{l}32,9 \% \\
(n=73)\end{array}$ & $\begin{array}{l}6,9 \% \\
(n=58)\end{array}$ & $\begin{array}{l}\mathrm{P}<10^{-3} ; \text { estadística } \\
\chi^{2} 12,98 ; \mathrm{gdl}=1\end{array}$ \\
\hline Consumo de otros - vida & $\begin{array}{l}23,3 \% \\
(n=73)\end{array}$ & $\begin{array}{l}12,1 \% \\
(n=58)\end{array}$ & $\begin{array}{l}P=0,099 ; \text { estadística } \\
\chi^{2} 2,72 ; \text { gdl }=1\end{array}$ \\
\hline
\end{tabular}

en la Región Metropolitana y no se concentraba necesariamente en las zonas aledañas al Instituto Psiquiátrico.

En relación a los 60 sujetos controles, su edad promedio era de 23 años, que era mayor que los pacientes $(\mathrm{P}<0,0002)$.

La exposición a cannabis en algún momento de la vida fue común en ambos grupos, con $87,8 \%$ en los jóvenes hospitalizados y $85 \%$ en controles $\left(\mathrm{p}=0,63 \chi^{2}\right.$; Tabla 2). Esto correspondía a un odds ratio de 1,27 (95\% IC 0,47-3,44; Figura 2), o controlado por edad y sexo de 1,51 (95\% IC $0,52-4,4)$. Al considerar la exposición regular (al menos 1 vez al mes), los jóvenes hospitalizados reportaron un período de consumo regular de 14 meses de mediana, en comparación con una mediana de 3 meses para los controles $(\mathrm{p}<0,023$, Mann-Whitney U test). La historia de un consumo regular de cannabis por más de 6 meses se relacionó con un odds ratio de 2,4 (95\% IC 1,14-5,05, Figura 2), corregido por edad y sexo de 2,05 (95\% IC 0,93-4,55).

En relación a la edad de primera exposición a cannabis, esta fue significativamente menor en los pacientes que en los controles (mediana de 16 años frente a 17, respectivamente, $\mathrm{p}<0,004$; Mann-Whitney U test). Otra forma de plantearlo es el odds ratio de psicosis asociado a consumo antes de los 16 años, que era de 3,85 (95\% IC 1,65-

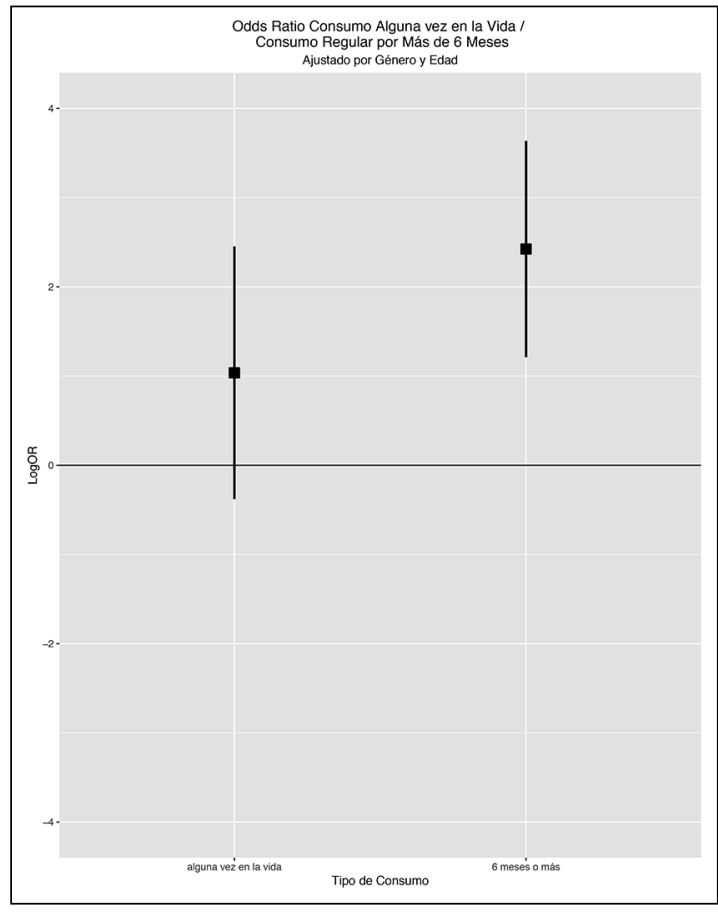

Figura 2. Odds ratio para la presencia de psicosis entre personas que han consumido marihuana alguna vez en su vida vs. aquellos que nunca han consumido, y odds ratio para la presencia de psicosis entre personas que han consumido marihuana de forma regular por al menos 6 meses versus aquellos que no han consumido o han consumido durante menos tiempo. Visualizado en escala logarítmica. 


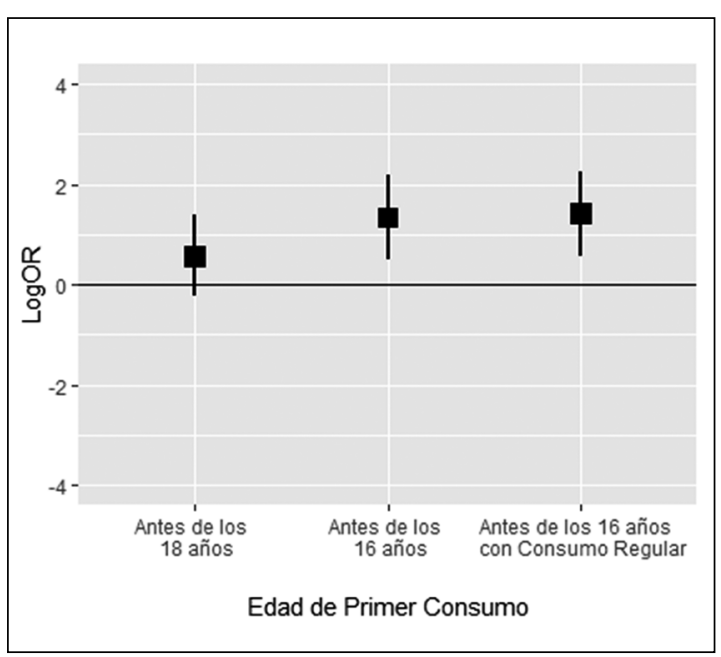

Figura 3. Odds ratio para presencia de psicosis entre personas que han consumido marihuana por primera vez antes de los 18 años versus aquellos que consumieron más tarde, para personas que han consumido marihuana antes de los 16 años versus aquellos que consumieron más tarde, y para personas que comenzaron a consumir marihuana de forma regular y antes de los 16 años versus aquellos que consumieron más tarde y/o con menor frecuencia. Visualizado en escala logarítmica.

8,99, Figura 3; ajustado por edad y sexo $1,96,95 \%$ IC 0,77-4,98). La asociación entre presencia de psicosis y consumo de cannabis solo al considerar el momento del primer consumo antes de los 18 años era ligeramente menor que el más temprano $(1,78,95 \%$ IC $0,79-4,03$; ajustado por edad y sexo $0,86,95 \%$ IC $0,35-2,13)$.

La asociación entre presencia de psicosis y consumo temprano y continuo de cannabis se vio más claramente al considerar ambos juntos: el OR de psicosis asociado a consumo antes de los 16 años y de forma regular por al menos 6 meses era de 4,18 (95\% IC 1,79-9,6; ajustado por edad y sexo 2,64, 95\% IC 1,08-6,57). La fracción atribuible poblacional a una historia de consumo regular con exposición temprana antes de los 16 años fue de 19,7\% (1,2-45,5\%).

Los pacientes también reportaron una historia de exposición a pasta base o cocaína, así como otras drogas, más alta que los controles, aunque solo para pasta base y cocaína fue estadísticamente significativo (Tabla 2). La asociación de historia de consumo regular de cannabis y psicosis se mantuvo en la dirección reportada al controlar por el consumo de estas otras sustancias en vida, aunque perdía significancia (OR 1,86; IC de 0,75 a 4,65, ajustado por edad, sexo, y consumo en vida de otras sustancias).De forma similar, la asociación entre consumo regular de inicio temprano y psicosis pierde fuerza al controlar por estos factores (OR 1,35; 0,62 a 2,98).

\section{Discusión}

Varios estudios internacionales previos han mostrado que cannabis es un factor de riesgo para psicosis en jóvenes de forma consistente. Los resultados de este estudio realizado con jóvenes hospitalizados por un primer episodio psicótico en Santiago de Chile sugieren que esto también aplica en nuestro país. Esta asociación es aun más clara en aquellos pacientes que reportan un consumo regular, empezado desde más temprana edad. Esto es consistente con los hallazgos en la literatura ${ }^{6}$.

El consumo de cannabis fue frecuente, tanto en nuestros pacientes como en los controles; $85 \%$ de este último grupo reportó haber consumido al menos una vez en la vida, y más de la mitad $(58,3 \%)$ declaró un consumo regular por más de 6 meses. Esta alta prevalencia del consumo de marihuana en la población general está en línea con los resultados presentados por el Servicio Nacional para la Prevención y Rehabilitación del Consumo de Drogas y Alcohol (SENDA), que reportan hasta $33,8 \%$ de consumo en jóvenes del grupo etario similar al de nuestros pacientes (19-25 años) ${ }^{3}$.

Nuestros pacientes también presentaron una exposición más temprana al consumo. Este es otro aspecto fundamental, ya que la literatura publicada lo identifica también como un factor de riesgo importante para presentar psicosis ${ }^{23,24}$. El consumo frecuente y desde más temprana edad se relacionó con un odds ratio de 4,18 veces, aunque este bajaba al ajustarlo por las diferencias demográficas entre los grupos a 2,6. Aun considerando este valor menor, el riesgo atribuible fue alrededor de $20 \%$. En términos concretos, esto sugiere que aproximadamente en 1 de cada 5 pacientes estaría asociada la presencia de psicosis con el contacto regular y temprano con cannabis. La magnitud de este dato hace que sea relevante para las políticas públicas, y resalta un área importante de prevención que tendría un efecto concreto en la salud de nuestros jóvenes. 
De acuerdo a nuestro conocimiento, si bien no existen estudios sobre la composición del cannabis en Chile, si contamos con reportes del SENDA y del Observatorio de Narcotráfico de la Fiscalía Nacional respecto a la irrupción, desde el año 2016, de marihuana creepy y su composición con alto contenido de THC, siendo actualmente el tipo de marihuana que cuenta con mayor oferta en el territorio nacional ${ }^{25}$.

A pesar de la escasa información sobre la composición de cannabis que actualmente se consume en Santiago, nuestros datos indican que el consumo regular de esta se asocia a la presencia de psicosis. A la luz de estos datos y considerando la tendencia alcista en el consumo de cannabis de los últimos años, se trataría de un problema de salud pública significativo.

Un confundente importante encontrado en nuestro estudio es el consumo de otras sustancias. Los pacientes reportaban una historia de uso (al menos una vez) de otras drogas de forma más frecuente que los controles, sobretodo cocaína o pasta base. Al controlar por este otro consumo se perdía la fuerza de la asociación entre cannabis y psicosis, no siendo ya significativa, aunque no se eliminaba del todo. Otros estudios han mostrado una asociación entre consumo de cocaína o sus derivados y psicosis ${ }^{17}$. Sin embargo, el control en el análisis del consumo de esta sustancia en otros estudios ha mantenido el efecto observado para cannabis ${ }^{26}$. Considerando que el modo de actuar de cannabis y cocaína no es el mismo en el cerebro, es posible que ambas drogas aumenten el riesgo de psicosis por mecanismos diferentes. La alta prevalencia del uso de cannabis, bastante mayor que la de cocaína o derivados, implica que su impacto en la sociedad es mayor. De ahí la importancia de resaltar sus efectos en el público general.

\section{Conclusión}

El consumo de cannabis fue frecuente en este grupo de jóvenes estudiados. Se asoció a la presencia de psicosis en este grupo de jóvenes hospitalizados por un primer episodio psicótico. Esta asociación es mayor cuando la edad de exposición a cannabis es más temprana y cuando la duración del consumo regular es mayor. Esto tiene consecuencias para la planificación de políticas de salud y en la prevención del consumo de cannabis en nuestra población, con especial énfasis en nuestros jóvenes.

\section{Limitaciones}

Como todo estudio caso control, nuestro estudio podría ser criticado por la selección de los controles. Estos eran algo mayores que los pacientes y con una mayor proporción de mujeres (esta última diferencia no fue significativa). La literatura muestra que los patrones de consumo de drogas varían entre la adolescencia y adulte $z^{27}$. Incluimos edad y género dentro del análisis como una forma de controlar por estas diferencias, manteniéndose significativo nuestro resultado principal sobre el aumento de riesgo por el consumo regular y temprano. Aun más, los controles se obtuvieron de círculos sociales de los mismos pacientes, lo que permitió parear por otras variables sociodemográficas. Por otro lado, no controlamos por consumo de alcohol u otras drogas. Por último, los estudios con diseño caso-control solo pueden mostrar asociaciones entre variables o fenómenos y no causalidad. Cabe considerar que la literatura previa ha reportado que la administración aguda puede causar psicosis ${ }^{11}$, así como el efecto dosis-respuesta encontrada con mayor consumo en la vida, datos que apoyan fuertemente una relación causal.

Agradecimientos: Este estudio fue realizado, en parte, gracias al apoyo de la Corporación Nacional de Ciencia y Tecnología (CONICYT), a través de su Programa de Estudio Interdisciplinario Anillo PIA ACT1414, FONDECYT regular 1160736 (NAC, JU), 1180358 (NAC, JU y AGV), el fondo de Investigación de Clínica Alemana de Santiago (JU), y de la Universidad FinisTerrae (RN, AGV).

\section{Referencias}

1. Nugent SM, Morasco BJ, O’Neil ME, Freeman M, Low A, Kondo K, Elven C, et al. The Effects of Cannabis among Adults with Chronic Pain and an Overview of General Harms: A Systematic Review. Ann Intern Med 2017; 167 (5): 319-31. Disponible en https://annals.org/ aim [Consultado el 18 de enero de 2019].

2. Whiting PF, Wolff RF, Deshpande S, Di Nisio M, Duffy S, Hernandez AV, et al. Cannabinoids for Medical Use: A Systematic Review and Meta-analysis. JAMA 2015; 
313 (24): 2456-73. Disponible en https://www.jamanetwork.com [Consultado el 18 de enero de 2019].

3. Servicio Nacional para la Prevención y Rehabilitación del Consumo de Drogas y Alcohol (SENDA). Décimo Segundo Estudio Nacional de Drogas en Población General, 2016. ISBN: 978-956-9141-54-6. Disponible en www.senda.gob.cl [Consultado el 25 octubre de 2018].

4. Instituto Nacional de la Juventud (INJUV). Octava Encuesta Nacional de Juventud 2015. 2017. ISBN: 978-956763624-2. Disponible en www.injuv.gob.cl [Consultado el 14 de mayo de 2019].

5. Andréasson S, Engström A, Allebeck P, Rydberg U. Cannabis and Schizophrenia: A Longitudinal Study of Swedish Conscripts. Lancet Psychiatry 1987; 330 (8574): 1483-6. Disponible en https://www.thelancet.com [Consultado el 25 de octubre de 2018].

6. Marconi A, Di Forti M, Lewis CM, Murray RM, Vassos E. Meta-analysis of the Association Between the Level of Cannabis Use and Risk of Psychosis. Schizophr Bull 2016; 42 (5): 1262-9. Disponible en https://academic. oup.com/schizophreniabulletin [Consultado el 14 de mayo de 2019].

7. Ferdinand RF, Sondeijker F, van der Ende J, Selten JP, Huizink A, Verhulst FC. Cannabis use predicts future psychotic symptoms, and vice versa.Addiction 2005; 100 (5): 612-8. Disponible en https://onlinelibrary.wiley.com [Consultado el 18 de enero de 2019].

8. Manrique-Garcia E, Zammit S, Dalman C, Hemmingsson T, Andreasson S, Allebeck P. Cannabis, schizophrenia and other non-affective psychoses: 35 years of follow-up of a population-based cohort. Psychol Med 2012; 42 (6): 1321-8. Disponible en https://www.cambridge.org [Consultado el 25 de octubre de 2018].

9. Colizzi M, Bhattacharyya S. Chapter 7-Neurocognitive effects of cannabis: Lessons learned from human experimental studies. ProgrBrain Res 2018; 42: 179-216. Disponibleenhttps://www.sciencedirect.com [Consultado el 18 de enero de 2019].

10. Vadhan NP, Corcoran CM, Bedi G, Keilp, JG, Haney M. Acute effects of smoked marijuana in marijuana smokers at clinical high-risk for psychosis: A preliminary study. Psychiatry Res 2017; 257 (11): 372-4. Disponible en https://www.sciencedirect.com [Consultado el 22 de agosto de 2019].

11. Murray RM, Englund A, Abi-Dargham A, Lewis DA, Di Forti M, Davies C, et al. Cannabis-associated psychosis: Neural substrate and clinical impact. Neuropharmacology 2017; 124 (9): 89-104. Disponibleenhttps://www.sciencedirect.com [Consultado el 22 de agosto de 2019].
12. McGuire P, Robson P, Cubala WJ, Vasile D, Morrison PD, Barron R, et al. Cannabidiol (CBD) as an Adjunctive Therapy in Schizophrenia: A multicenter Randomized Controlled Trial. Am J Psychiatry 2018; 175 (3): 225-31. Disponible en https://ajp.psychiatryonline.org [Consultado el 28 de agosto de 2019].

13. Fusar-Poli P, Crippa JA, Bhattacharyya S, Borgwardt SJ, Allen P, Martin-Santos R, et al. Distinct Effects of $\Delta$ 9-Tetrahydrocannabinol and Cannabidiolon Neural Activation During Emotional Processing. Arch Gen Psychiatry 2009; 66 (1): 95-105. Disponible en https:// jamanetwork.com [Consultado el 20 de diciembre de 2018].

14. Di Forti M, Marconi A, Carra E, Fraietta S, Trotta A, Bonomo $\mathrm{M}$, et al. Proportion of patients in south London with first-episode psychosis attributable to use of high potency cannabis: a case-control study. Lancet Psychiatry 2015; 2 (3): 233-8. Disponible en https:// www.thelancet.com [Consultado el 21 de noviembre de 2018].

15. Freeman TP, Winstock AR. Examining the profile of high-potency cannabis and its association with severity of cannabis dependence. Psychol Med 2015; 45 (15): 3181-9. Disponible en https://www.ncbi.nlm.nih.gov/ pmc/articles/PMC4611354/[Consultado el 21 de noviembre de 2018].

16. Di Forti M, Quattrone D, Freeman T, Tripoli G, Gayer-Anderson C, Quigley $\mathrm{H}$, et al. The Contribution of Cannabis Use to Variation in the Incidence of Psychotic Disorder across Europe (EU-GEI): A multicentre case-control study. Lancet Psychiatry 2019; 6 (5): 427-36. Disponible en https:/www.thelancet.com/journals/ lanpsy/article/PIIS2215-0366(19)30048-3/fulltext [Consultado el 28 de agosto de 2019].

17. Libuy N, de Angel V, Ibáñez C, Murray RM, Mundt AP. The relative prevalence of schizophrenia among cannabis and cocaine users attending addiction services. Schizophr Res 2018; 194 (4): 13-7. Disponible en https:/www.sciencedirect.com/science/article/abs/pii/ S0920996417301974 [Consultado el 13 de febrero de 2019].

18. Programa de Intervención Temprana en Psicosis. Instituto Psiquiátrico Dr. José Horwitz Barak. Disponible en https://www.proitp.cl/ [Consultado el 19 de diciembre de 2018].

19. Sheehan DV, Lecrubier Y, Sheehan KH, Amorim P, Janavs J, Weiller E, et al. The mini-international neuropsychiatric interview (M.I.N.I.): the development and validation of a structure diagnostic psychiatric interview for DSM-IV and ICD-10. J Clin Psychiatry 1998; 59 (20): 22-33; quiz 34-57. Disponible en https://www.psy- 
chiatrist.com/jcp/article/Pages/1998/v59s20/v59s2005. aspx [Consultado el 12 de septiembre de 2018].

20. Barkus EJ, Stirling J, Hopkins RS, Lewis S. Cannabis-induced psychosis-like experiences are associated with high schizotypy. Psychopathology 2006; 39 (4): 175-8. Disponible en https://www.karger.com/Article/ Abstract/92678 [Consultado el 16 de abril de 2020].

21. Tripepi G, Jager KJ, Dekker FW, Zoccali C. Stratification for Confounding - Part 1: The Mantel-Haenszel Formula. Nephron ClinPract 2010; 116 (4): c317-c321. Disponible en https://www.karger.com/Article/FullText/319590 [Consultado el 15 de noviembre de 2018].

22. Llorca J, Fariñas-Álvarez C, Delgado-Rodríguez M. Fracción Atribuible Poblacional: Cálculo e interpretación. Gaceta Sanitaria 2001; 15 (1): 61-7. Disponibleenhttps://www.sciencedirect.com/science/article/ pii/S0213911101715191 [Consultado el 15 de octubre de 2018].

23. Helle S, Ringen PA, Melle I, Larsen TK, Gjestad R, Johnsen E, et al. Cannabis use is associated with 3years earlier onset of schizophrenia spectrum disorder in a naturalistic, multi-site sample ( $\mathrm{N}=1119)$, Schizophr Res. 2016; 170 (1): 217-21. Disponible en https://www.sciencedirect.com/science/article/pii/S0920996415300700 [Consultado el 22 de octubre de 2018].

24. Donoghue K, Doody GA, Murray RM, Jones PB, Mor- gan C, Dazzan P, et al. Cannabis use, gender and age of onset of schizophrenia: Data from the ÆSOP study. Psychiatry Res 2014; 215 (3): 528-32. Disponible en https://www.sciencedirect.com/science/article/abs/pii/ S0165178113008329 [Consultado el 09 de noviembre de 2018].

25. Unidad Especializada en Tráficoilícito de Estupefacientes y Sustancias Sicotrópicas. Informe 2018. Observatoriodel narcotráfico en Chile. Disponible en http://www.fiscaliadechile.cl/observatoriodrogaschile/ informe_2018_online.html [Consultado el 07 de marzo de 2019].

26. Moore TH, Zammit S, Lingford-Hughes A, Barnes TR, Jones $\mathrm{PB}$, Burke $\mathrm{M}$, et al. Cannabis use and risk of psychotic or affective mental health outcomes: a systematic review. Lancet Psychiatry 2007; 370 (9584): 319-28. Disponible en https:/www.thelancet.com/journals/lancet/ article/PIIS0140-6736(07)61162-3/fulltext [Consultado el 16 de abril de 2020].

27. Staff J, Schulenberg JE, Maslowsky J, Bachman JG, O'Malley PM, Maggs JL, et al. Substance use changes and social role transitions: proximal developmental effects on ongoing trajectories from late adolescence through early adulthood. Dev Psychopathol 2010; 22 (4): 917-32. Disponible en https://pubmed.ncbi.nlm. nih.gov/20883590/ [Consultado el 16 de abril de 2020]. 